\title{
The Characteristics of Brain Structural Network in Patients with Low Grade Glioma Revealed by Diffusion Tensor Imaging
}

\author{
Ling Tao ${ }^{1 *}$, Zhiyu Qian ${ }^{1}$, Yamin Yang ${ }^{1}$, Hongyi Liü ${ }^{2}$, and Li Xue ${ }^{1}$ \\ ${ }^{1}$ Nanjing University of Aeronautics and Astronautics, No.29, Yudao Street, Nanjing, 210016, China \\ ${ }^{2}$ Nanjing Brain Hospital Affiliated to Nanjing Medical University, No.264, Guangzhou Road, Nanjing, 210029, China \\ *e-mail: taoling@nuaa.edu.cn
}

\begin{abstract}
Purpose: At present, few studies have investigated the functional performance in patients with low grade glioma (LGG) from the perspective of brain structural characteristics. This study aimed to analyze the topological properties of brain structural networks in LGG patients and investigate the influence of operation and lesion location on whole-brain and hemisphere networks. On this basis, the structural plasticity and function compensatory mechanism of LGG patient were discussed. Method: We constructed whole-brain and hemisphere anatomical networks for 20 LGG patients and 20 normal controls (NC). The structural connections were established by selecting an appropriate connectivity threshold. Brain hub nodes and random nodes between LGG and NC were compared. Statistical analyses were performed to reveal the significant differences of various network characteristics between LGG patients and NC as well as between LGG patients before and after operation. The two-factor variance analysis was used to investigate the influence of operation and lesion location on wholebrain and hemisphere networks. Results: From the graph-based topological metrics of the constructed network, various global parameters changed significantly in LGG patients. Meanwhile, altered regions in the LGG patients was obtained and various nodal parameters were further calculated in each region. Most of hub nodes were identical between LGG and NC groups, while the betweenness centrality values of those hub nodes and some random nodes were higher in the LGG patients. By comparing LGG patients pre- and post- operation, more significantly altered network metrics were obtained from hemisphere network analysis than that from whole-brain network analysis, and network features of dominant hemisphere changed more drastically when the lesion is located in the same hemisphere. Conclusion: To conclude, the present study indicates the existence of compensatory mechanism in LGG patients to adapt to cognitive requirements. Function reorganization and the rewiring of neuronal circuits allow signal transmission bypassing the lesion area or surgical trauma. It also suggests that graph-based topological metrics analysis could become a useful method to provide valuable indices of brain function in the evaluation of the LGG. (C) 2017 Journal of Biomedical Photonics \& Engineering.
\end{abstract}

Keywords: Structural network; Topological properties; Brain plasticity; Compensatory mechanism; Functional lateralization.

Paper \#3154 received 24 Jan 2017; revised manuscript received 5 May 2017; accepted for publication 10 May 2017; published online 15 Jun 2017. doi: 10.18287/JBPE17.03.030301. 


\section{References}

1. E. G. Shaw, B. W. Scheithauer, and J. R. OFallon, "Management of supratentorial low-grade gliomas," Seminars in Radiation Oncology 1(1), 23-31 (1993).

2. E. F. Chang, A. Clark, J. S. Smith, M.-Y. Polley, S. M. Chang, N. M. Barbaro, A. T. Parsa, M. W. McDermott, and M. S. Berger, "Functional mapping-guided resection of lowgrade gliomas in eloquent areas of the brain: improvement of long-term survival," Journal of Neurosurgery 114(3), 566-573 (2011).

3. J. R. Petrella, L. M. Shah, K. M. Harris, A. H. Friedman, T. M. George, J. H. Sampson, J. S. Pekala, and J. T. Voyvodic, "Preoperative functional MR imaging localization of language and motor areas: effect on therapeutic decision making in patients with potentially resectable brain tumors," Radiology 240(3), 793-802 (2006).

4. S. M. Smirnakis, M. C. Schmid, B. Weber, A. S. Tolias, M. Augath, and N. K. Logothetis, "Spatial specificity of BOLD versus cerebral blood volume fMRI for mapping cortical organization," Journal of Cerebral Blood Flow \& Metabolism 27(6), 1248-1261 (2007).

5. A. G. Goloshevsky, C. W.-H. Wu, S. J. Dodd, and A. P. Koretsky, "Mapping cortical representations of the rodent forepaw and hindpaw with BOLD fMRI reveals two spatial boundaries," Neuroimage 57(2), 526-538 (2011).

6. C. M. Filley (ed.), The Behavioral Neurology of White Matter, Oxford University Press, New York (2001). ISBN: 978-0-1951-3561-9.

7. P. J. Basser, and D. K. Jones, "Diffusion-tensor MRI: theory, experimental design and data analysis - a technical review," NMR Biomed 15(7-8), 456-467 (2002).

8. E. Bullmore, and O. Sporns, "Complex brain networks: graph theoretical analysis of structural and functional systems," Nature Reviews Neuroscience 10(3), 186-198 (2009).

9. M. R. Del Bigio, M. J. Wilson, and T. Enno, "Chronic hydrocephalus in rats and humans: white matter loss and behavior changes," Ann Neurol 53(3), 337-346 (2003).

10. Y. Ding, J. P. McAllister, B. Yao, N. Yan, and A. I. Canady, "Axonal damage associated with enlargement of ventricles during hydrocephalus: a silver impregnation study," Neurol Res 23(6), $581-587$ (2001).

11. M. Lazar, A. L. Alexander, P. J. Thottakara, B. Badie, and A. S. Field, "White matter reorganization after surgical resection of brain tumors and vascular malformations," American journal of neuroradiology 27(6), 1258-1271 (2006).

12. G. Gong, Y. He, L. Concha, C. Lebel, D. W. Gross, A. C. Evans, and C. Beaulieu, "Mapping anatomical connectivity patterns of human cerebral cortex using in vivo diffusion tensor imaging tractography," Cerebral cortex 19(3), 524-536 (2009).

13. B. Wang, Y. Fan, M. Lu, S. Li, Z. Song, X. Peng, R. Zhang, Q. Lin, Y. He, J. Wang, and R. Huang, "Brain anatomical networks in world class gymnasts: A DTI tractography study," NeuroImage 65, 476-487 (2013).

14. J. T. Duda, P. A. Cook, and J. C. Gee, "Reproducibility of graph metrics of human brain structural networks," Frontiers in Neuroinformatics 8(46), (2014).

15. M. Bastiani, N. J. Shah, R. Goebel, and A. Roebroeck, "Human cortical connectome reconstruction from diffusion weighted MRI: the effect of tractography algorithm," NeuroImage 62(3), 1732-1749 (2012).

16. C. Thomas, F. Q. Ye, M. O. Irfanoglu, P. Modi, K. S. Saleem, D. A. Leopold, and C. Pierpaoli, "Anatomical accuracy of brain connections derived from diffusion MRI tractography is inherently limited," Proceedings of the National Academy of Sciences 111(46), 16574-16579 (2014).

17. B. C. M. van Wijk, C. J. Stam, and A. Daffertshofer, "Comparing brain networks of different size and connectivity density using graph theory," PLoS One 5(10), e13701 (2010).

18. D. J. Watts, and S. H. Strogatz, "Collective dynamics of 'small-world' networks," Nature 393(6684), 440-442 (1998).

19. M. D. Humphries, and K. Gurney, "Network 'small-world-ness': a quantitative method for determining canonical network equivalence," PLoS One 3(4), e0002051 (2008).

20. O. Sporns, and J. D. Zwi, "The small world of the cerebral cortex," Neuroinformatics 2(2), 145-162 (2004).

21. R. Albert, and A. Barabási, "Statistical mechanics of complex networks," Reviews of modern physics 74(1), 47-97 (2002).

22. S. Achard, and E. Bullmore, "Efficiency and cost of economical brain functional networks," PLoS Comput Biol 3(2), e17 (2007).

23. L. C. Freeman, "A set of measures of centrality based on betweenness," Sociometry 40(1), 35-41 (1977).

24. V. Gol'dshtein, G. A. Koganov, and G. I. Surdutovich, "Vulnerability and hierarchy of complex networks," arXiv preprint, cond-mat/0409298 (2004).

25. P. Holme, B. J. Kim, C. N. Yoon, and S. K. Han, "Attack vulnerability of complex networks," Physical Review E 65(5), 1-15 (2002).

26. L. da F. Costa, F. A. Rodrigues, G. Travieso, and P. R. Villas Boas, "Characterization of complex networks: A survey of measurements," Advances in Physics 56(1), 167-242 (2007). 
27. M. Girvan M, and M. E. J. Newman, "Community structure in social and biological networks," Proceedings of the National Academy of Sciences of the United States of America 99(12), 7821-7826 (2002).

28. V. Latora, and M. Marchiori, "Efficient behavior of small-world networks," Physical Review Letters 87(19), 1-4 (2001).

29. M. Filippi, and F. Agosta, "Magnetic resonance techniques to quantify tissue damage, tissue repair, and functional cortical reorganization in multiple sclerosis," Prog Brain Res 175, 465-482 (2009).

30. C. Sampaio-Baptista, A. A. Khrapitchev, S. Foxley, T. Schlagheck, J. Scholz, S. Jbabdi, G. C. DeLuca, K. L. Miller, A. Taylor, N. Thomas, J. Kleim, N. R. Sibson, D. Bannerman, and H. Johansen-Berg, "Motor skill learning induces changes in white matter microstructure and myelination, "J Neurosci 33(50), 19499-19503 (2013)

31. S. R. Wainwright, and L. A. M. Galea, "The Neural Plasticity Theory of Depression: Assessing the Roles of Adult Neurogenesis and PSA-NCAM within the Hippocampus,” Neural Plasticity 2013, 1-14 (2013).

32. Healthline Medical Team, Frontal lobe, Medically Reviewed, 2 March 2015.

33. M. Macmillan, "Alfred walter campbell and the visual functions of the occipital cortex," Cortex 56, 157-181 (2014).

34. E. J. J. Habets, A. Kloet, R. Walchenbach, C. J. Vecht, M. Klein, and M. J. B. Taphoorn, “Tumour and surgery effects on cognitive functioning in high-grade glioma patients," Acta Neurochirurgica 156(8), 1451-1459 (2014).

35. M. Klein, H. Duffau, and P. C. D. W. Hamer, "Cognition and resective surgery for diffuse infiltrative glioma: an overview," Journal of neuro-oncology 108(2), 309-318 (2012).

36. K. Caeyenberghs, and A. Leemans, "Hemispheric lateralization of topological organization in structural brain networks," Human brain mapping 35(9), 4944-4957 (2014).

37. H. Cheng, Y. Wang, J. Sheng, W. G. Kronenberger, V. P. Mathews, T. A. Hummer, and A. J. Saykin, "Characteristics and variability of structural networks derived from diffusion tensor imaging," Neuroimage 61(4), 1153-1164 (2012).

38. D. K. Jones, T. R. Knosche, and R. Turner, "White matter integrity, fiber count, and other fallacies: the do's and don'ts of diffusion MRI," Neuroimage 73, 239-254 (2013).

H. Huang, N. Shu, V. Mishra, T. Jeon, L. Chalak, Z. J. Wang, N. Rollins, G. Gong, H. Cheng, Y. Peng, Q. Dong, and Y. He, "Development of Human Brain Structural Networks Through Infancy and Childhood," Cerebral Cortex 25(5), 1389-1404 (2015).

\section{Introduction}

Low-grade gliomas (LGGs) are a diverse group of gliomas that arise from uncontrolled growth of brain glial cells [1]. Surgical resection is considered as the primary option for the clinical treatment of LGG, with the aim of maximum tumor removal while preserving functional cortical and subcortical areas so as to minimize postoperative morbidities. The gold standard to identify the brain tumor margins and important brain regions is intraoperative electrophysiological cortical mapping (ECM), which has already been verified for its clinical application in maximizing tumor resection and significantly improving long-term survival in LGG patients [2]. However, intraoperative ECM often requires active participation and compliance of patients, high demands of technical expertise and is highly time and effort consuming.

In recent years, blood oxygenation level dependence functional MRI (BOLD-fMRI) has demonstrated its particular value in preoperative risk assessment in patients with LGG, which can facilitate planning of surgery, shorten the duration of the operation, and obtain prognostic information prior to surgery [3]. Nevertheless, BOLD-fMRI only provides information on cortical representation of brain function, but not on the course of the subcortical and deep white matter tracts $[4,5]$. As the white matter possesses about $50 \%$ of the adult brain volume and consists of a complex array of neuronal fiber connectivity [6], brain lesions often affect the white matter and may alter the known anatomical path in which the fiber pass. Inadvertent transection may lead to equally devastating results as the resection of the eloquent cortex. As to LGG patients, functional impairment is not only originated from tumor areas, but also could be affected by damaged fiber bundles that connect tumor lesions with the rest of the brain. In order to assess the structural integrity and fiber orientation, diffusion tensor imaging (DTI) has been developed as a noninvasive MRI technique to provide information about the integrity and physiology of white matter fiber tracts and neural pathway connections [7].

Recently, network model has been proposed as a useful tool for investigating the structural organization and functional mechanisms of the human brain. Graph theoretical analysis can reveal key topological and geometrical properties of network such as smallworldness, modularity, and highly connected hubs, heterogeneous degree distributions, network information transfer efficiency and so on. Graph theoretical approaches can provide a new powerful way of quantifying the brain's structural and functional systems, identifying the alteration of brain activation and localizing corresponding functional regions in patients [8]. In contrast to the large number of studies 
on functional network regarding other brain diseases, there are only a handful of reports on examining the function areas from the perspective of the cerebral anatomical network, and little is known about the alteration of the white matter fiber connection patterns in LGG patients.

Permanent damage to white matter integrity is known to occur in cases of severe and chronic exposure to mechanical intra-cranial pressure as happens in hydrocephalus $[9,10]$. In case of LGG, the lesion mostly causes the fiber displacement or temporary damage which may result in substantial reorganization of the functional areas and could be recovered after operation [11]. Therefore, understanding the characteristics of brain anatomical network in LGG patients and how structural changes may affect the functional integration and interaction between different regions is very necessary for presurgical planning and evaluation of surgical treatments.

In present study, we constructed the whole brain and hemisphere anatomical networks for 20 brain LGG patients and 20 healthy subjects based on DTI technique, and then various graph theoretical analysis approaches were applied to examine the topological properties of brain network. Statistical analysis was performed to determine group differences in global and nodal properties between LGG patients and normal controls as well as between pre-surgery patients and post-surgery patients. Furthermore, the two-factor variance analysis was used to investigate whether the surgical treatment has an influence on hemisphere networks and whether the topological properties of hemisphere networks would change significantly when the lesion located in different brain hemisphere. The possible change of white matter plasticity and brain functional lateralization were discussed based on above results.

\section{Methods and Materials}

\subsection{Subjects}

This study included 20 LGG patients (10 males; mean age 51 years; range 16-67 years) and 20 healthy adult subjects (10 males; mean age 25 years; range $24-34$ years). All patients with LGG were selected from Nanjing Brain Hospital, among which 12 cases of lesions are in the right hemisphere and 8 cases of lesions are in the left hemisphere. All LGG patients were diagnosed as frontal lobe or temporal lobe tumor, and the histopathological diagnosis was performed according to the WHO Classification of tumors affecting the central nervous system. All healthy subjects as the normal controls (NC) were recruited from voluntary participants in university. All participants were right-handed and had no history of neurological or psychiatric disease or head injury. All LGG patients received clinical cognitive function assessment pre-operation and post-operation, and had no significant cognitive dysfunction. Each participant was provided with a written informed consent before DTI examinations and this study was approved by the Medical Research Ethics Committee of Nanjing Brain Hospital.

\subsection{Image acquisition}

DTI was performed with a $3 \mathrm{~T}$ Siemens Trio MR system with a 32-channel phased-array head coil. Head motion was minimized with restraining foam pads provided by the manufacturer. DTI data were acquired by employing a single-shot twice-refocused echo planar imaging (EPI) sequence in alignment with the anterior-posterior commissural plane. The parameters used for diffusion data acquisition were as follows: repetition time $(\mathrm{TR})=6,500 \mathrm{~ms}$, echo time $(\mathrm{TE})=95 \mathrm{~ms}, 30$ nonlinear diffusion directions with $\mathrm{b}=1000 \mathrm{~s} / \mathrm{mm}^{2}$ and an additional volume with $\mathrm{b}=0 \mathrm{~s} / \mathrm{mm}^{2}$, acquisition matrix $=128 \times 128$, field of view $($ FOV $)=240 \mathrm{~mm} \times 240 \mathrm{~mm}$, bandwidth $(\mathrm{BW})=1447 \mathrm{~Hz} /$ pixel. $3 \mathrm{D}$ high-resolution brain structural images (voxel size $=1 \mathrm{~mm}^{3}$, isotropic) were acquired using a T1-weighted magnetizationprepared rapid gradient-echo (MP-RAGE) sequence for each subject. The sequence parameters: TR/TE = $1900 \mathrm{~ms} / 2.49 \mathrm{~ms}$, inversion time (TI) $=900 \mathrm{~ms}$, flip angle $(\mathrm{FA})=9^{\circ}, \mathrm{FOV}=250 \mathrm{~mm} \times 250 \mathrm{~mm}$, and 176 sagittal slices covering the whole brain. For each subject, both the DTI data and the brain structural images were acquired in the same session.

\subsection{Data preprocessing and network construction}

Firstly, the cerebrum was automatically partitioned into 90 cortical and subcortical regionals (45 for each hemisphere) according to the widely accepted Automated Anatomical Labeling (AAL) atlas [12]. Since this atlas only has T1-weighted structural images, the DTI image cannot be directly registered to the AAL atlas. Therefore, inverse transformations were used to warp the AAL atlas from the Montreal Neurological Institute (MNI) space to the DTI native space in which the discrete labeling values were preserved by using a nearest neighbor interpolation method [12, 13]. Briefly, for each subject, the individual T1-weighted images were firstly coregistered to the b0 images in the native diffusion space using a linear transformation. The transformed $\mathrm{T} 1$ images were then nonlinearly transformed to the ICBM152 T1 template in MNI space. The above linear and nonlinear transformations were all performed using the SPM8 package (http://www.fil.ion.ucl.ac.uk/spm/software/spm8).

Using this procedure, we obtained 90 cortical and subcortical region of interest (ROIs), each representing a node of the network.

Next, the fibers linking each pair of brain regions in the diffusion space were reconstructed. The whole-brain fiber tracking was constructed using DTI studio2.4 software (http://www.mristudio.org) based on the Fiber Assignment by Continuous Tracking (FACT) algorithm. Fiber tracking was stopped at voxels where FA $<0.2$ or 
if the turning angle between two eigenvectors of two consecutive voxels was greater than $45^{\circ}$ [13].

To define the network edges, we selected a threshold value of three fibers to ensure that the average size of the biggest connected component of the network keeps 90 across all subjects (threshold selection will be introduced in details in section 2.4). That is to say, if there are at least three fibers with end points in regions $u$ and $v$, then the two nodes $u$ and $v$ are connected with an edge. And the number of fibers between regions was only used to indicate the existence/absence of the edge. In this way, the binarized anatomical network for each subject was constructed and represented by a symmetric $90 \times 90$ matrix.

The construction process of anatomical networks is illustrated in Fig. 1. Firstly, AAL atlas in DTI native space (Fig. 1 (a)) was used to segment the whole-brain fiber tracking results (Fig. 1 (b)) into 90 regions. Then, fiber number in each region was extracted, and a $90 \times$ 90 binary matrix (Fig. 1 (c)) was obtained by setting a threshold value of fiber number. Next, according to the symmetric $90 \times 90$ matrix, we can extract two $45 \times 45$ binary matrix representing the structural connectivity of left and right hemisphere, respectively (Fig. 1 (e) and (f)). The visualization of brain networks (Fig. 1 (d), (g) and (h)) were obtained using Brainnet software (http://www.nitrc.org/projects/bnv/).

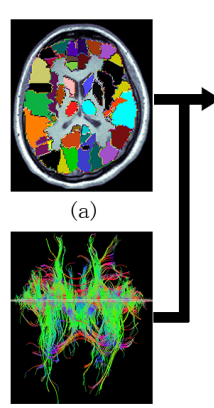

(b)

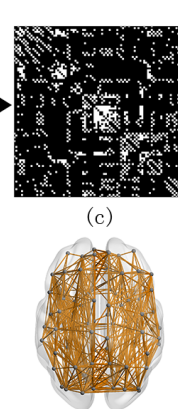

(d)

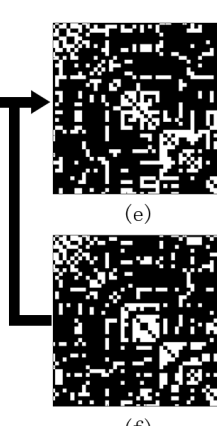

(f)

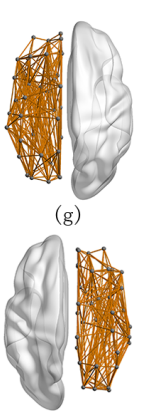

(h)
Fig. 1 Schematic illustration of the construction process of structural networks by DTI. (a) The AAL atlas was warped from the MNI space to the DTI native space by using inverse transformations, obtaining the parcellation of the cortex into 90 brain regions for each subject. (b) The whole-brain fiber tracts were reconstructed by using deterministic fiber tractography. (c) A $90 \times 90$ binary matrix was obtained by extracting fiber number in each region. (d) The visualization of whole-brain anatomical network. A $45 \times 45$ binary matrix representing the structural connectivity of (e) left and (f) right hemisphere. The visualization of $(\mathrm{g})$ left and $(\mathrm{h})$ right hemisphere anatomical network.

\subsection{Threshold selecting}

During brain network construction, the network matrix threshold $(\tau)$ is critical, and the optimal value of $\tau$ would result in the most accurate reconstruction of networks [14]. Brain networks, as well as their topological properties, are often sensitive to the connectivity threshold [15-16]. If threshold $\tau$ is too low, the resulting network would include connections that do not actually exist in reality and vice versa. Even a small number of spurious or misdetected edges can affect the properties of networks to a large extent [17]. In our study, we determined the optimal threshold value based on the small-world characteristics of network. The concept of small-world was originally proposed by Watts and Strogatz [18]. The small-world properties of a network can be characterized by the normalized clustering coefficient $(\gamma)$ and the normalized characteristic path length $(\lambda)$ of the network. These two conditions can be summarized into a scalar quantitative measurement, small-worldness, $\sigma=\gamma / \lambda$, which is typically larger than 1 for small-world networks [18, 19]. Many studies revealed that human brain has a typical small-world topology, which is characterized by large clustering coefficients and short mean path length $[18,20]$.

In this study, we evaluated the effects of different network matrix thresholds on the small world characteristics by setting the number of fiber bundles ranged from 1 to 5 (Fig. 2). We found that small worldness reached maximum when the fiber number was set to be 3 , and thus the threshold of 3 fibers was used for anatomical networks construction in Section 2.3 .

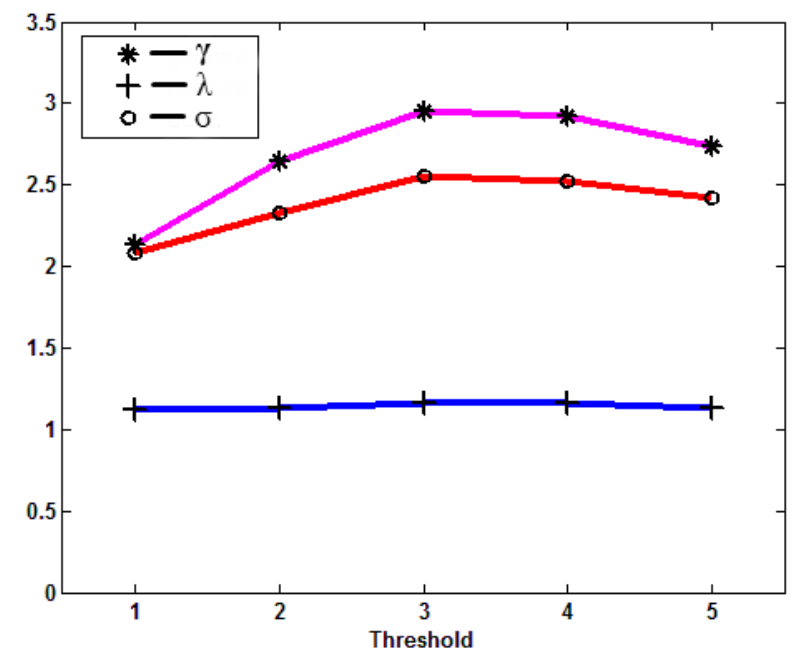

Fig. 2 Small world parameters under different threshold.

\subsection{Network Analysis}

The topological properties of anatomical networks were investigated at the global and regional (nodal) levels, respectively. The global properties of the whole brain networks were quantified in terms of clustering coefficient $\left(C_{p}\right)$, characteristic path length $\left(L_{p}\right)$, degree $\left(K_{p}\right)$, local efficiency $\left(E_{l o c}\right)$ and global efficiency $\left(E_{\text {glob }}\right)$ [19-20]. The regional properties were investigated by analyzing degree $\left(K_{i}\right)$, clustering coefficient $\left(C_{i}\right)$, shortest path length $\left(L_{i}\right)$, local efficiency ( $\left.E_{i_{-} \text {local }}\right)$, regional efficiency $\left(E_{\text {nodal }}\right)$, 
normalized betweenness centrality $\left(b_{i}\right)$ and vulnerability $\left(V_{i}\right)$ of the node $i$ [21-24]. Based on the constructed anatomical network of each subject, significant differences in global and the nodal properties between LGG patient and NC group were further studied.

As an important parameter of network evaluation, betweenness centrality is often used to assess the hub nodes in complex networks. The node with higher betweenness centrality value often exists in the concentrated areas with shortest path length, which means that more information will pass through and converge at this node. In this study, we calculated the value of betweenness centrality of 90 regions, and the averaged value of each region in all 20 subjects within the same group.

The vulnerability of the network can also be used to quantitatively assess the damage on network performance caused by a simulated failure of its element, e.g. removal of a particular node [24]. If one wants to protect the network by guarding or by a temporary isolation of some nodes (edges), the most important nodes (edges), breaking of which makes the whole network malfunctioning, should be identified [25]. In our study, to calculate the vulnerability values $\left(V_{i}\right)$ of each node and edge, we removed the nodes or edges one by one from the network and calculated the changes in global efficiency of resulting network by: $V_{i}=\left(E_{\text {glob }}-E_{\text {glob }}^{\prime}\right) / E_{\text {glob }}$. Where $E_{\text {glob }}^{\prime}$ is the global efficiency after the removal of the node $i$ and all its edges [26].

\subsection{Statistical analysis}

Based on the binary network construction result in each subject, between-group differences (NC and LGG groups) in the graph-based metrics (global parameters $C_{p}, L_{p}, K_{p}, E_{l o c}, E_{g l o b}, \gamma, \lambda$, and $\sigma$ ) of the anatomical networks were tested by two-sample t-tests. A two-sample two-tailed t-test was used to detect the relationship between nodal properties in network metrics with significant group difference, and a paired- samples $t$ test was used to detect statistical difference between pre-operation and post-operation groups.

\section{Results}

\subsection{Altered topological properties of anatomical networks in $L G G$ patients}

From the graph-based topological metrics of the constructed network, various global parameters $\left(C_{p}\right.$, $L_{p}, K_{p}, E_{l o c}, E_{g l o b}, \gamma, \lambda$, and $\sigma$ ) were calculated in all three groups, i.e. NC, pre-operation LGG and postoperation LGG group, and between-group statistical comparison was performed to detect significant difference, respectively. As shown in Table 1-3, all of three groups have similar path lengths $(\lambda \approx 1)$ and high local clustering coefficients $(\gamma>>1)$, exhibiting typical small-world features.

Statistical analysis indicated that, compared with NC group, there is a significant increase in normalized clustering coefficient $(\gamma)(p=0.037$ and $p=0.036)$, small-worldness $(\sigma)(p=0.043$ and $p=0.040)$, and characteristic path length $\left(L_{p}\right)(p=0.037$ and $p=0.035)$ in the anatomical networks of LGG patients (both pre-operation and post-operation), while a significant lower degree $\left(K_{p}\right)(p=0.022$ and $p=0.025)$ and global efficiency $\left(E_{\text {glob }}\right)(p=0.030$ and $p=0.032)$ were found in LGG patients. In addition, aside from the characteristic path length $\left(L_{p}\right)(p=0.048)$, there is no significant statistical difference in most of topological metrics of the whole-brain network in LGG patients preand post-operation. Therefore, following analysis of whole-brain topological properties will only be performed between NC and pre-operation LGG groups. As to the post-operation LGG group, the corresponding effects on the network properties upon surgical treatments will be discussed in details by deciphering the topological metrics of hemisphere networks.

Table 1 The comparison of topological properties between NC and pre-operation LGG groups.

\begin{tabular}{lllllllll} 
& $\gamma^{*}$ & $\lambda$ & $\sigma^{*}$ & CP & LP* & KP* & Eglob* & \\
\hline NC & 2.6837 & 1.1422 & 2.3496 & 0.4953 & 2.3115 & 12.8706 & 0.5022 \\
\hline LGG(pre-) & 2.8472 & 1.1531 & 2.4692 & 0.4950 & 2.3801 & 11.9356 & 0.4871
\end{tabular}

*Significant group differences at $\mathrm{p}<0.05$

Table 2 The comparison of topological properties between NC and post-operation LGG groups.

\begin{tabular}{|c|c|c|c|c|c|c|c|c|}
\hline & $\gamma^{*}$ & $\lambda$ & $\sigma^{*}$ & $\mathrm{CP}$ & $\mathrm{LP}^{*}$ & $\mathrm{KP}^{*}$ & Eglob* & Eloc \\
\hline $\mathrm{NC}$ & 2.6837 & 1.1422 & 2.3496 & 0.4953 & 2.3115 & 12.8706 & 0.5022 & 0.7452 \\
\hline LGG(post-) & 2.8511 & 1.1536 & 2.4712 & 0.4951 & 2.4212 & 11.8701 & 0.4827 & 0.7473 \\
\hline
\end{tabular}

*Significant group differences at $\mathrm{p}<0.05$. 
Table 3 The comparison of topological properties between pre- and post-operation LGG groups.

\begin{tabular}{lllllllll} 
& $\gamma$ & $\lambda$ & $\sigma$ & $\mathrm{CP}$ & LP* & KP & Eglob & Eloc \\
\hline LGG(pre-) & 2.8472 & 1.1531 & 2.4692 & 0.4950 & 2.3801 & 11.9356 & 0.4871 & 0.7434 \\
\hline LGG(post-) & 2.8511 & 1.1536 & 2.4712 & 0.4951 & 2.4212 & 11.8701 & 0.4827 & 0.7473
\end{tabular}

*Significant group differences at $\mathrm{p}<0.05$

\subsection{Alteration of hub nodes in patients}

Betweenness centrality and vulnerability were used to define network hub nodes. Firstly, the average betweenness centrality and vulnerability of each node in $\mathrm{NC}$ and LGG groups were calculated, respectively. As shown in Fig.3, the betweenness centrality values of 90 brain regions were sorted in descending order for $\mathrm{NC}$ group, and the betweenness centrality values for LGG groups and the vulnerability values for both two groups were sorted accordingly. On the whole, the changes of betweenness centrality values coincided with the changes of vulnerability values in corresponding groups. As the betweenness centrality of nodes are closely related to the network performance, and the removal of nodes with higher betweenness centrality would result in possible damage of network, i.e. the increase of vulnerability values. Consistent with previous studies, nodes with higher betweenness centrality value are thus identified as hub nodes in our study [23, 27]. More specifically, a node was defined as hub node if its betweenness centrality value is at least one standard deviation above the network mean. Thirteen hub nodes were determined in NC group, among which 9 hub nodes locating in associative cortex, 2 hub nodes locating in paralimbic cortex, and 2 hub nodes locating in subcortical region. In LGG group, 10 hub nodes were identified, including 5 hub nodes in associative cortex, 3 hub nodes in paralimbic cortex, and 2 hub nodes in subcortical region.

In order to visualize the location of hub nodes, 3D distribution diagram of hub nodes was obtained (Fig. 4). Clearly, most of the hub nodes were identical and distributed in same location (with color-coded in red) in both NC and LGG group, while 3 hub regions only existed in the NC group (middle part in Fig. 4, colorcoded in green), i.e. left superior frontal gyrusdorsolateral (SFGdor.L), left superior occipital gyrus (SOG.L) and left Lingual gyrus (LING.L)In order to show the similarities in the spatial patterns of the node betweenness of the two groups, we plotted the correlation-degree of the betweenness centrality of all 90 regions in the anatomical networks of the $\mathrm{NC}$ and LGG groups. As shown in Fig. 4 (right), red dots represent hub regions that exist in both groups, green dots refer to the 3 hub regions specific to the NC group, and pink circles represent those nodes that are not identified as hub nodes. From our results, the non-hub nodes (random nodes) showed the best correlation between the two groups $(r=0.8907, p=7.13 e-32)$, which means the majority of structural networks may remain relatively stable in LGG group. On the other hand, some random nodes with increased betweenness centrality values were found in LGG group, which could contribute to the alteration of brain functional network.

\subsection{Alteration of network regional parameters in patients}

The topological properties of anatomical networks were also investigated at regional (nodal) levels. Altered regions in the LGG patients was obtained and various nodal parameters were further calculated in each region. Two-sample two-tailed t-test was performed to determine group difference in the nodal properties between the NC and LGG groups. Three significance thresholds of $p<0.05,0.01$, and 0.005 was used for testing each of the graph-based metrics, respectively. As shown in Fig. 5, compared with NC group, significant decrease of degree $\left(K_{i}\right)$ and regional efficiency $\left(E_{\text {nodal }}\right)$ were found in LGG group in various regions, especially in the frontal (ORBsupmed.L, ORBinf.L, ORBsup.L, SFGdor.L), occipital (SOG.R), supplementary motor areas (SMA.L, SMA.R) and median cingulate and paracingulate gyri (DCG). On the contrary, path length $\left(L_{i}\right)$ increased significantly in the frontal and occipital regions, while demonstrating opposite changes in the supplementary motor areas and median cingulate and paracingulate gyri. The clustering coefficient $\left(C_{i}\right)$ and local efficiency $\left(E_{i_{-} \text {local }}\right)$ showed a significant decrease in the middle frontal gyrus (ORBmid.L) and a significant increase in the olfactory cortex (OLF) in LGG patients.

\subsection{Altered topological properties of hemisphere networks in patients}

In order to investigate whether the topological properties of hemisphere networks (lesion side) would have significant changes when the lesion located in different brain hemisphere, and whether the operation (tumor removal) has an influence on hemisphere networks (lesion side), we performed the two-factor variance analysis with "Lesion location" and "surgery or not" as the fixed factors. Variance analysis results in Table 4 indicated that, surgery would result in significant changes in various parameters including $K_{p} 、 L_{p} 、 E_{\text {glob }}$, while the lesion location in different brain hemisphere would only cause significant difference in clustering coefficient $\left(C_{p}\right)$. 

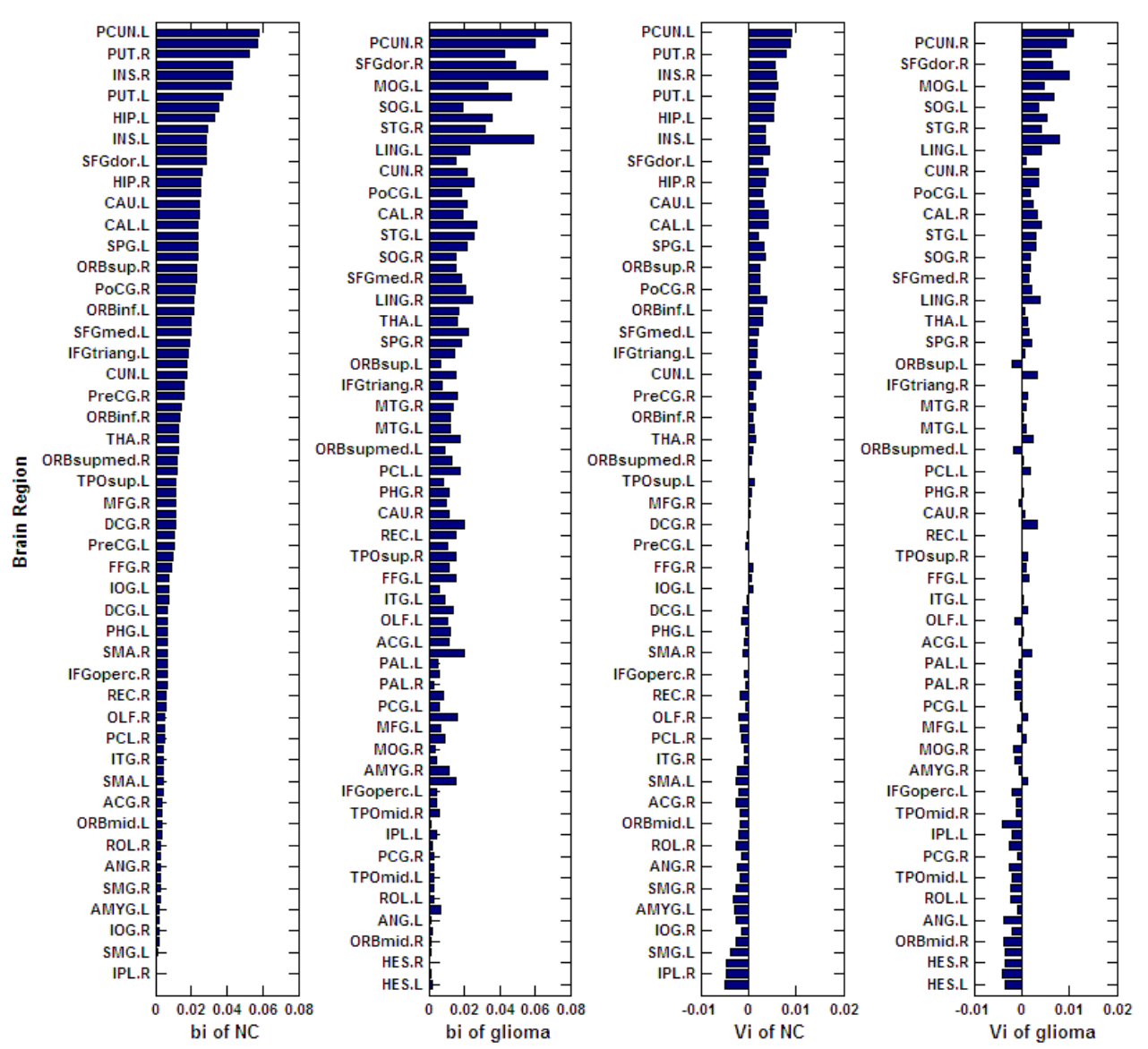

Fig. 3 Betweenness centrality (bi) and vulnerability (Vi) values of corresponding brain regions in NC and LGG groups.
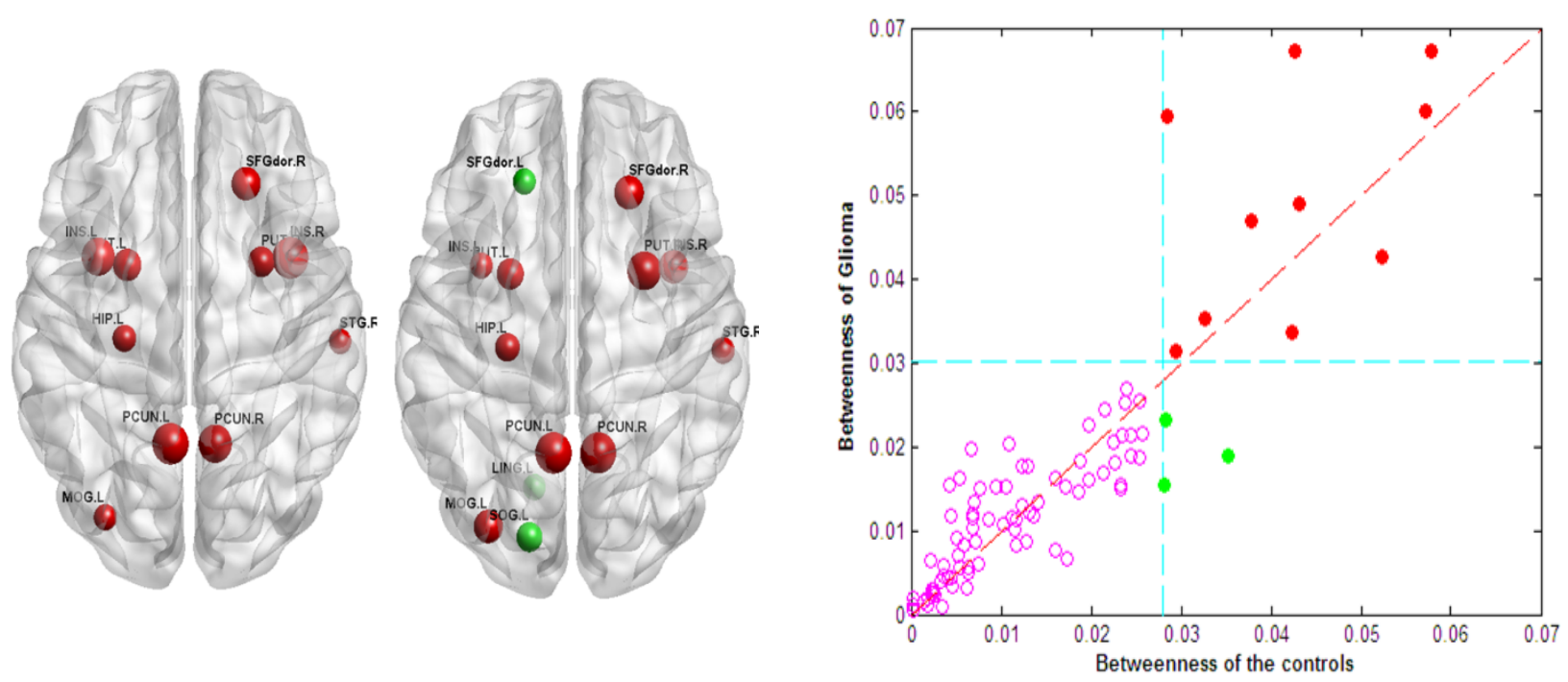

Fig. 4 Hub nodes distribution and correlation degree of all nodes between NC and LGG groups. From left to right: hub nodes distribution in LGG group; hub nodes distribution in NC group; the correlation-degree of betweenness centrality of all nodes between two groups. The size of the node represents the magnitude of the betweenness centrality. Nodes color-coded in red represent hub regions detected in the anatomical networks of both the subject groups. Nodes colorcoded in green represent hub regions specific to the controls. The location of the regions was visualized with the BrainNet View software (http://www.nitrc.org/projects/bnv). 

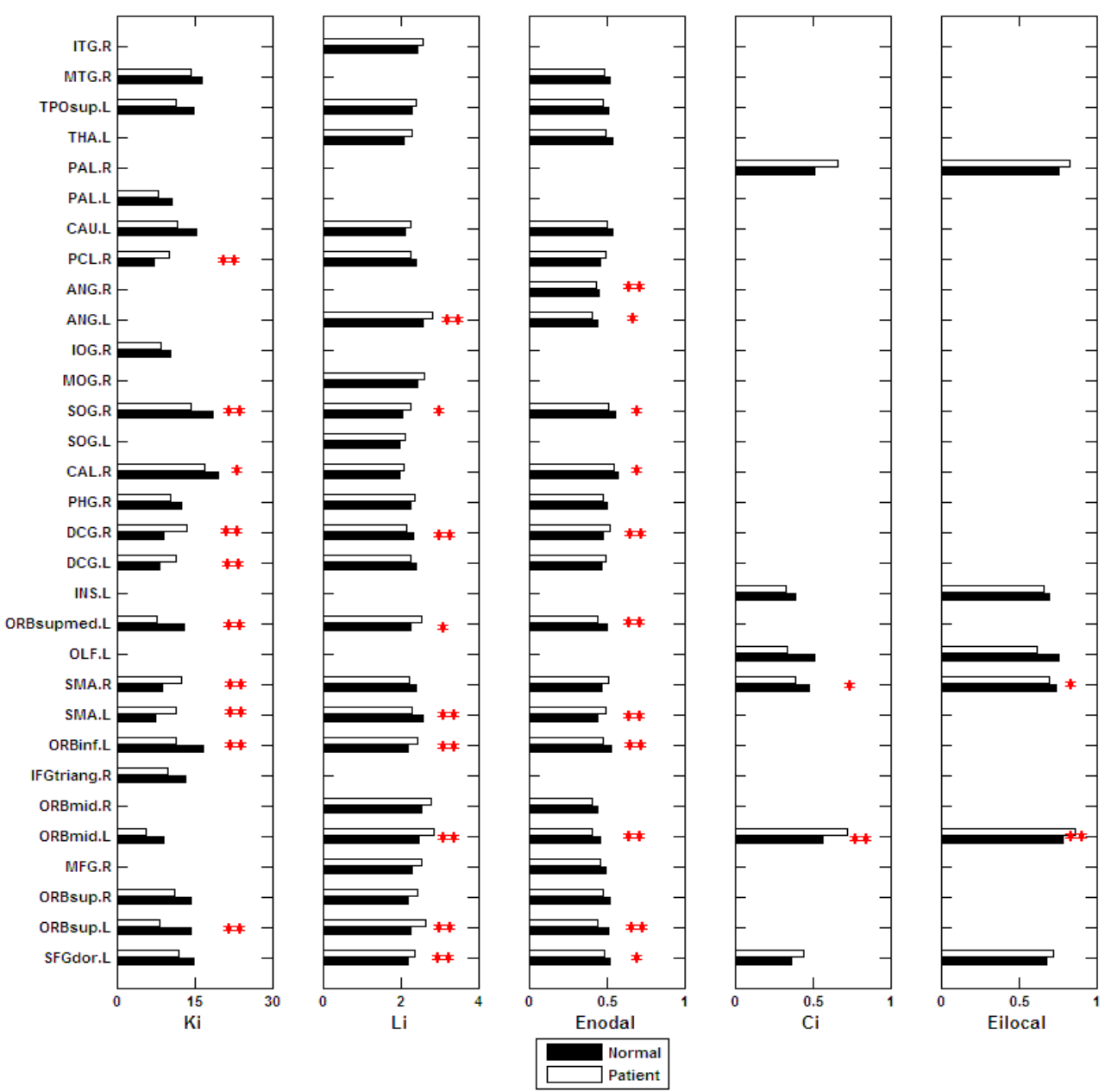

Fig. 5 Distribution of regions with significantly altered nodal properties in NC and LGG groups. Unstarred bars: significance with $\mathrm{p}<0.05 ; *: \mathrm{p}<0.01 ; * *: \mathrm{p}<0.005$.

Table 4 The results of variance analysis with "Lesion location" and "surgery or not" as the fixed factors.

\begin{tabular}{llcccccccc} 
& & $K_{p}$ & $C_{p}$ & $L_{p}$ & $E_{\text {glob }}$ & $E_{\text {local }}$ & $\gamma$ & $\lambda$ & $\sigma$ \\
\hline \multirow{2}{*}{ Lesion(left/right) } & $\mathrm{F}$ & 0.559 & 4.189 & 0.027 & 0.153 & 3.521 & 1.215 & 0.112 & 1.531 \\
\cline { 2 - 9 } & $\mathrm{P}$ & 0.350 & $0.040^{*}$ & 0.917 & 0.712 & 0.083 & 0.282 & 0.751 & 0.233 \\
\hline \multirow{2}{*}{ Surgery(pre-/post-) } & $\mathrm{F}$ & 5.287 & 0.128 & 5.512 & 5.519 & 0.076 & 2.899 & 2.181 & 2.745 \\
\cline { 2 - 9 } & $\mathrm{P}$ & $0.023^{*}$ & 0.744 & $0.026^{*}$ & $0.021^{*}$ & 0.813 & 0.095 & 0.162 & 0.111
\end{tabular}

Note: Significant group differences at $* \mathrm{p}<0.05$

Table 5 The parameter comparison of hemisphere networks with significant difference.

\begin{tabular}{llllll} 
& $E_{\text {glob }}$ & $K_{p}$ & $L_{p}$ & pre $-C_{p}$ & post $-C_{p}$ \\
\hline Pre- & 0.572 & 11.214 & 2.321 & - & - \\
\hline Post- & 0.559 & 10.387 & 2.386 & - & - \\
\hline left & - & - & - & 0.532 & 0.538 \\
\hline right & - & - & - & 0.579 & 0.545
\end{tabular}

Note: Significant group differences at $* \mathrm{p}<0.05$ 
Comparing those network parameters $\left(K_{p} 、 L_{p}\right.$ 、 $\left.E_{g l o b}\right)$ with significant difference between preoperation and post-operation groups, we found that the value of global efficiency $\left(E_{g l o b}\right)$ and degree $\left(K_{p}\right)$ decreased, while the characteristic path length $\left(L_{p}\right)$ increased after surgery (Table 5). In addition, regardless of operation conditions, for the lesion in right hemisphere, the clustering coefficient $\left(C_{p}\right)$ were always greater than that in left hemisphere.

\section{Discussion}

\subsection{Altered topological properties of anatomical networks in patients}

This study compared the global and regional properties of brain anatomical networks of LGG patients with those of healthy controls. Consistent with previous studies, we found that the anatomical networks of both the LGG patients (pre- and post-surgery) and the controls showed features of small-worldness, characterized by a high value of local clustering coefficients $(\gamma>>1)$ but low values of path lengths $(\lambda \approx 1)$.

Based on the graph theoretical analysis results, a decrease in degree and global efficiency and an increase in path length were found in LGG group. As the degree of each node is closely related to the connectivity of anatomical network, a decreased degree in LGG patients could result in a sparser network as compared with $\mathrm{NC}$ group [19]. According to the definition of key metrics in graph theory, the decreased degree will increase the path length of signal flow, and will contribute to the decrease of global efficiency [28]. In good agreement with this, decreased global efficiency and increased path length were indeed found in LGG patients, which is suggestive of slow information transmission and inefficient communication between brain regions. Interestingly, our graph analysis also revealed changes in the small-world network architecture, with a significant increase in small-worldness and clustering coefficient in LGG patients. More prominent small world network organization was observed in LGG patients compared to healthy controls, which could be interpreted as a compensatory mechanism. Comparing LGG patients pre- and post- operation, there is no significant between-group difference in small-world characteristics despite an increased path length in postsurgery group. As the topological characteristics of whole-brain network remained almost stable regardless of surgery conditions, we speculate that local network properties could be better indicators for evaluating the surgical effectiveness and will discuss in details below.

\subsection{Alteration of hub nodes in patients.}

Compared with normal subjects, three hub nodes were missing in LGG patients: left superior frontal gyrus- dorsolateral (SFGdor.L), left superior occipital gyrus (SOG.L) and left Lingual gyrus (LING.L). SOG.L and LING.L are both located in the upper part of occipital lobe, and SFGdor.L is located in the middle-upper part of frontal gyrus, suggesting these brain regions could be more susceptible to LGG. The absence of these hub nodes indicates that the topological structure of functional networks was probably restrained by the lesions in LGG patients, and these regions could become less important for information transmission with the presence of LGG.

The distribution of other hub nodes, such as precuneus (PCUN), insula (INS), lenticular putamen (PUT), hippocampus (HIP), and the pillow back (MOG), are mostly identical between NC and LGG group. However, the betweenness centrality values of these hub nodes are higher in LGG group than those in $\mathrm{NC}$, suggesting these hub nodes could be directly associated with structural and functional modifications and become more important in the anatomical network in LGG group. Most of the hub nodes tend to change towards a high betweenness centrality value (red dots in Fig. 4 right) in LGG patients, while the majority of random nodes (non-hub nodes) remained stable between two groups. Nevertheless, there exist some random nodes with high-betweenness value, and these nodes may play more active role in the network due to brain plasticity and functional modifications. When signals cannot directly flow through the shortest path between two hub nodes, it may flow through indirect connections of non-hub nodes [29, 30]. In the human brain network, evidence suggests that mechanisms of brain plasticity include the strengthening of specific connections or the recruitment of parallel and indirect connections [31]. Therefore, based on this mechanism, the rewiring of neuronal circuits and reorganization of tracts between the regions may occur in response to LGG, allowing signal transmission via longer paths bypassing the lesion area. Indeed, in our results, significantly increased average path length was found in the LGG group.

It also implies that, for presurgery planning, we cannot only focus on the hub nodes, and those random nodes with temporary function alteration and neural reconnection capabilities may also play critical roles in the anatomical network. We therefore further investigated the function alteration by analyzing regional parameters of anatomical networks.

\subsection{Alteration of network regional parameters in patients}

Based on the analysis results of regional parameters, the node degrees and node efficiency of the LGG patients showed a significant decrease, while the average path length demonstrated a significant increase in the frontal and occipital regions. Furthermore, the clustering coefficient and local efficiency of the LGG patients also have a significant decrease in the frontal region. These finds suggest that the frontal region could be severely 
damaged, which can be mainly explained by the presence of LGG in the frontal lobe or temporal areas in the subjects. The function of the frontal lobe involves integrating voluntary movement, longer non-task based memories, and accessing information for analysis and judgment [32]. Theoretically, the changes of regional parameters could be indicative of corresponding functional impairment. However, in our study, all of LGG patients were reported with no obvious clinical cognitive deficits, and we thus hypothesize that there may be some recovery on the basis of compensation mechanism as well as the participation of other regions to assume the lost functions of damaged areas. As the occipital regions are mainly responsible for visual functions [33], great alteration of nodal parameters found in occipital regions is consistent with clinical reports of vision loss in LGG patients. In addition, there were a significant increase of clustering coefficient and local efficiency in the supplementary motor area (SMA) and median cingulate and paracingulate gyri (DCG) in LGG patients, which indicated that these two regions might have more connections with other brain regions and play emerging roles in information signal transfer. In fact, SMA and DCG were not determined as hub nodes, but they could become more important during functional compensation due to brain structural plasticity. Together with above random nodes analysis results, in the presence of LGG lesions, function reorganization could exist with involvement of random nodes. During presurgical planning, while special attention should be paid into more accurate identification of the few highly influential nodes, the functions of those non-highly influential nodes (non-hub nodes) should never be underestimated. A throughout analysis of the regional parameters of brain network would help us to determine the critical nodal areas which could be related to possible functional impairment, so as to avoid unwanted damage during surgery and preserve the brain function to the maximum extent.

\subsection{Altered topological properties of hemisphere networks in the patients}

By comparing the topological properties of whole-brain network in LGG patients before and after operation, characteristic path length is the only parameter that changed significantly. In contrast, the hemisphere network metrics showed a great dependence on the operation, and the degree, global efficiency and the shortest path greatly altered with significant difference between pre- and post-surgery patients. We thus assume that local damage caused by operation could contribute to the altered network properties of affected brain hemisphere, while the whole-brain function could be compensated due to possible tracts reorganization and self-repairing mechanism.

Previous researches have showed that cognitive impairment may have significant difference when the lesion is located on different brain hemispheres.
Compared with patients with gliomas located in nondominant hemisphere, the patients with gliomas located in the advantage hemisphere usually had more severe cognitive impairment [34]. Moreover, resections of dominant temporal lobe were reported to be correlated with verbal memory decline, whereas non-dominant temporal lobe resections were associated with visuospatial memory decline [35]. From our results, the clustering coefficient showed a great dependence on the lesion location. As all of our subjects are right-handed and the left hemisphere is usually considered as the dominant hemisphere, brain network features of left hemisphere could be more sensitive to lesion sites and surgical trauma, which can be explained by the smaller cluster coefficient found in the group with lesion in left hemisphere. The asymmetry characteristics of brain network in healthy individual is associated with cognitive differences between left and right hemisphere [36]. Clearly, studies regarding network topological properties of hemispheres could bring more insights in understanding the lesion location-related brain cognitive damage and provide more knowledge for the evaluation of surgical treatment.

\subsection{Methodological issues}

Several methodological issues related to present study are discussed below. Firstly, in consideration of its simplicity, robustness and speed, and for better comparison with other previously reported studies [37], the commonly used deterministic tractography algorithm was applied to track fiber in our study. Noticed that this method cannot resolve crossing fiber bundles [38], future study using probabilistic tractography [39] could be an alternative approach to address this issue. Secondly, there is actually no physiological criterion for definition edge and node selection in human brain anatomical network construction. Similar to other studies [12-13], we used the AAL template and fiber number to determine nodes and edges, respectively. However, as the nodes and edges are closely related to the substantial analysis results of the constructed network, their physiological definition could be of great importance and requires future studies supported by a wealth of evidence from both anatomical and physiological studies. At last, as a pilot proof-of-concept study, the results obtained in our studies were based on a relatively small sample size. We believe that more comprehensive investigation towards a more precise data interpretation could be achieved with continuous expanding of sample size.

\section{Conclusion}

In this paper, we constructed the anatomical networks of whole-brain and hemisphere in $\mathrm{NC}$, pre-surgery and post-surgery LGG patient groups, then the topological properties and regional parameters of networks were analyzed and compared, respectively. Our results showed that the presence of lesion and operation may alter the information transmission path between regions, 
making original direct signal transmission through hub nodes turn into indirect signal transmission through non-hub nodes, characterized by the increase of path length. At the same time, more prominent small world network organization in LGG patients can evidence the active processes of neuroplasticity and compensatory mechanism in response to the presence of LGG. In addition, due to possible tract reorganization and network reconfiguration, some non-highly influential nodes became progressively important with the presence of lesion or in response to operation, of which functions cannot be underestimated. It is thus necessary to investigate those nodal regions by further analyzing regional parameters of network. Comparing the wholebrain and hemisphere anatomical networks of LGG patients pre- and post- operation, whole-brain function in post-operation LGG patients could be compensated as a result of self-repairing mechanism, while hemisphere network metrics changed significantly before and after operation, suggesting that hemispheredependent network analysis could bring valuable information for the evaluation of treatment effectiveness. Furthermore, it is found that brain network metrics altered more significantly when the lesion is located in the dominate hemisphere. This finding could be helpful for understanding the cognitive function related to lesion location and provide a new way to predict and evaluate the effects of surgical treatments.

\section{Acknowledgments}

This work was supported by the Natural Science Foundation of China (Grant numbers: 61075107,
81601532), Natural Science Foundation of Jiangsu Province (BK20160814), and the Clinical medical science Special Foundation in Jiangsu Province (Grant numbers: SBL201230215), and Scientific Research Foundation of Nanjing University of Aeronautics and Astronautics (1003-YAH16009). Ling Tao would also like to thank China Scholarship Council awarding me a scholarship to pursue study in Monash University as a visiting scholar.

\section{Confliance with ethical standards}

\section{Conflict of interes}

Ling Tao, Zhiyu Qian, Yamin Yang, Hongyi Liu, Li Xue declare that they have no conflict of interest.

\section{Ethical standard}

All procedures were followed in accordance with the ethical standards of the responsible committee on human experimentation (institutional and national) and with Declaration of the Helsinki 1975, as revised in 2008 (5).

\section{Informed consent}

Informed consent was obtained from all patients for being included in the study. 